\title{
Snacking patterns among adolescents: a comparison of type, frequency and portion size between Britain in 1997 and Northern Ireland in 2005
}

\author{
Maeve A. Kerr, Kirsten L. Rennie, Tracy A. McCaffrey, Julie M. W. Wallace, Mary P. Hannon-Fletcher \\ and M. Barbara E. Livingstone* \\ Northern Ireland Centre for Food and Health (NICHE), University of Ulster, Coleraine BT52 1SA, UK \\ (Received 7 December 2007 - Revised 3 April 2008 - Accepted 10 April 2008 - First published online 5 June 2008)
}

Despite the potential link between snack food intake and obesity and the reportedly high prevalence of snacking among adolescents, adolescent snack food patterns (types of foods consumed, frequency and portion size) have not been extensively examined. This study examines these issues using data on the snacking patterns of adolescents aged 13-16 years who took part in the 1997 National Diet and Nutrition Survey (NDNS) and that from a Northern Irish (NI) cohort of adolescents collected 8 years later, in 2005. Overall energy intake was significantly higher in the NI adolescents in 2005 compared with the NDNS adolescents in $1997(P<0 \cdot 01)$. Consequently, energy intake from snacks was significantly higher in the NI cohort $(P<0.01)$ and a trend for a higher \% energy intake from snacks compared with the NDNS group was observed (median $32.5 \%$ v. $29.8 \%$, respectively). Sugar-sweetened carbonated and soft drinks remained the most popular choice of snack over this 8-year period; however, both the portion size consumed and frequency of consumption were significantly higher among the adolescents in 2005 compared with those in 1997 ( $P=0.022$ and $P=0.014$, respectively). Despite the lower popularity, and correspondingly lower frequency of milks and beverages, the portion size of both food groups was significantly higher among the adolescents in 2005 compared with those in 1997 ( $P<0.001$ and $P=0.007$, respectively). These findings may provide scope for policy interventions to place particular emphasis on reducing typical portion sizes consumed of popular snack choices, in particular high-energy carbonated and soft drinks, among UK adolescents.

Snacking: Portion size: Energy intake: Adolescents

Obesity among children and adolescents is a major public health concern. It is predicted that, by 2025 , the prevalence of obesity among UK children and adolescents will have increased to $15 \%$ and will escalate to $25 \%$ by $2050^{(1)}$. The reported decline in the traditional habit of eating three meals per $d$ and an increase in 'snacking' has coincided with the rise in overweight and obesity among children and adolescents $^{(2)}$. Consequently, several studies have focused on a potential causal link between obesity and snack food intake. While there is still no direct evidence to confirm this association in either children or adults, both the energy density of specific foods commonly eaten as snacks and the frequency of snack food consumption have increased over the last few decades among young adults in the USA ${ }^{(3)}$. Furthermore, it has been reported that there may be no compensation made for the increased energy intake (EI) from energy-dense snacks at subsequent eating occasions ${ }^{(4)}$.

EI from snacks has increased by $30 \%$ among US children and adolescents in the last few decades, accounting for a quarter of total $\mathrm{EI}^{(5)}$. Such data are not surprising, given that popular snack food choices among US children and adolescents include energy-dense items such as cakes, cookies and savoury snacks ${ }^{(6)}$. Furthermore, portion sizes of popular snack food choices such as salty snacks and soft drinks were reported to have increased among US individuals aged 2 years and older from $1977-1998^{(7)}$. In a recent study among Finnish adults, foods typically consumed as snacks were reported to be higher in energy density than those consumed as meals and included sweet bakery goods, breads, sweets and chocolate, while foods typically consumed as main meals included meat and fish dishes, potatoes and cooked vegetables ${ }^{(8)}$. An earlier British study examining snack $v$. meal intakes within three different age groups (elderly, middle-aged and young adults) observed that, overall, the contributions of protein and fat to the energy content of snacks were lower than those of meals, while those derived from total sugars were greater ${ }^{(9)}$. Interestingly, it was reported in the latter study that adolescents consumed more of their total EI as snacks compared with young adults and the elderly (26\% compared with $19 \%$ and $17 \%$, respectively).

Despite the potential link between snack food intake and obesity and the reportedly high prevalence of snacking among adolescents, snack food patterns (in terms of types of foods consumed, frequency of consumption and portion size) and how they have evolved over time have not been extensively examined. Consequently, the aim of the current

Abbreviations: DLW, doubly labelled water; EER, estimated energy requirement; EI, energy intake; IQR, interquartile range; NDNS, National Diet and Nutrition Survey; NI, Northern Ireland; TEE, total energy expenditure.

* Corresponding author: Professor M. B. E. Livingstone, fax + 4428703 23023, email mbe.livingstone@ulster.ac.uk 
investigation was to examine these issues using data on the snacking patterns of adolescents aged 13-16 years who took part in the 1997 National Diet and Nutrition Survey (NDNS) and that from a Northern Irish (NI) cohort of adolescents collected 8 years later, in 2005 .

\section{Methods}

\section{National Diet and Nutrition Survey adolescents}

Data from the NDNS were obtained from the UK Data Archive, University of Essex. The NDNS of young people aged 4-18 years is part of a rolling programme of governmentcommissioned surveys of different age groups of the free-living population of Great Britain (England, Wales and Scotland). Details of the survey respondents and methods have been described in more detail elsewhere ${ }^{(10)}$. Briefly, a nationally representative sample of 2672 young people aged 4-18 years was randomly sampled from 132 postcode sectors throughout mainland Britain in 1997. Only young people living in private households were included and only one child per household was accepted. The survey design included an interview to provide information on socio-demographic circumstances of the young person's household, medication use and eating and drinking habits. The survey fieldwork was divided into four 3-month 'waves', between January 1997 and December 1997, therefore spanning a full calendar year. Ethical approval for the survey was obtained from National Health Services Local Research Ethics Committees.

\section{Northern Irish adolescents}

The second group of adolescents (NI cohort) in the current study were initially recruited through primary schools in the Coleraine area of NI between 1996 and 1998 when they were aged 6-8 years (baseline study) to participate in a study examining EI in relation to obesity risk. Full details of the study and procedures have been explained elsewhere ${ }^{(11)}$. In 2005, when the children were aged 13-16 years, fifty subjects $(44 \%)$ of the original cohort agreed to participate in the follow-up study. There were no significant differences between the participants who declined to take part in the follow-up and those who participated in both the baseline and the follow-up studies in terms of age, weight, height, BMI, total energy expenditure (TEE), EI:energy expenditure and dietary intake data (data not shown). Following the protocol for the baseline study, all follow-up measurements took place during the school term and were conducted over a 1-year period. At baseline and follow-up the parents of each subject gave written informed consent to their child's/adolescent's participation in the study. Ethical approval for the study was obtained from the Research Ethics Committee of the University of Ulster.

\section{Dietary intake data: National Diet and Nutrition Survey}

Of the sample of 2672 young people identified for the survey, a total of 2127 participants completed the interview, of which 1701 participants completed $7 \mathrm{~d}$ weighed dietary records. Of these, all dietary intake data collected from adolescents aged 13-16 years $(n$ 434) were selected for the current analysis, including one subject who reported they consumed no snacks over the $7 \mathrm{~d}$ recording period. Each young person or carer was supplied with a set of digital food scales and two recording diaries; the 'home record' diary for foods eaten in the home and the 'eating out' diary for foods that were eaten outside the home and could not be weighed. A description of each food or drink item consumed over the $7 \mathrm{~d}$ period was recorded, including the brand name of the food/drink and the method of preparation, if appropriate. The weight of food served, the weight of any leftovers and consumption of vitamin and mineral supplements were also recorded. When a served item could not be weighed, the young person and/or carer was asked to record a description of the portion size, using standard household measures. In addition, for each day of the recording period the young person and/or carer was asked to record whether illness during the recording period affected their eating and whether they were dieting to lose weight during the recording period. A feasibility study was conducted before the survey, in which the dietary record method was validated against doubly labelled water (DLW) measures of TEE for estimating EI ${ }^{(12)}$

\section{Dietary intake data: Northern Ireland cohort}

Of the fifty subjects who participated in the NI follow up study, two subjects failed to provide any dietary data and one subject did not provide complete dietary data, leaving a total sample of forty-seven adolescents for the current analysis. Briefly, adolescents were issued with digital weighing scales and instructed how to weigh and record all food and drinks consumed, as well as leftovers, for seven consecutive days. Researchers gave detailed explanations and demonstrated the cumulative weighing technique and then asked the adolescents to repeat the procedure in their presence. In addition, written instructions and an example of a complete diary were provided for reference inside the food diary. Subjects were asked to provide a complete description of the method of preparation and cooking and recipes for composite dishes; adjustments were made for fluid losses during cooking. Food eaten outside the home was identified by brand name and packet size or the empty wrappers. For foods that could not be weighed and not packaged, i.e. school lunches, subjects were asked to provide a detailed description of the foods eaten and to estimate the quantity consumed. At each home visit, the researcher checked these and added missing detail as appropriate. Food diaries were analysed using WISP (version 3.01; Tinuviel Software, UK).

\section{Definition of a snack}

In the NI cohort, the subjects were asked to self-define eating occasions (i.e. meal or snack); however, no self-definition of eating occasions was included in the NDNS dietary record. In order to make valid comparisons between snacks consumed by the NDNS adolescents and those by the NI adolescents, the same criteria to distinguish snacks from meals were applied to both cohorts. The criterion chosen was time of day. The time frames applied to the current study were broadly consistent with self-defined snack time frames among the NI adolescents. 
Meals were identified as eating events that took place within three specific time frames of 06.00 to 09.30 hours (breakfast), 11.30 to 14.30 hours (lunch) and 16.30 to 19.30 hours (dinner). All eating events that took place outside of these time frames were categorised as snacking occasions.

\section{Food group analysis}

Every food item recorded in the dietary record for the NDNS (1997) was allocated an individual food code and each food code was allocated to one of 115 subsidiary food groups. Food items recorded in the dietary record for the NI cohort (2005) were also allocated an individual food code and allocated to one of 147 food groups ${ }^{(13)}$. For the purposes of the current study, following selection of snacks only, the food groups in both the NDNS survey and the NI cohort were aggregated into the same twenty-eight main food groups. The twenty-eight food groups were subsequently ranked in descending order according to the proportion of subjects who consumed those food groups during the $7 \mathrm{~d}$ recording period. The top fifteen food groups in both studies were considered to be the most commonly consumed snack foods. Subsequent food group analysis was based on these top fifteen ranked snack foods among the NDNS adolescents. Average EI from snacks were calculated as total EI from snacks divided by the total number of days for which dietary intake was recorded: $7 \mathrm{~d}$ for all NDNS participants and ranging from $5-7 \mathrm{~d}$ for the NI cohort.

\section{Anthropometric measurements}

For the NDNS group, measurements of standing height were taken using the portable Leicester height measure. Weight was measured using the Soehnle Quantratronic digital personal weighing scales. For the NI cohort, body weight in a swimming costume was measured to the nearest $0.1 \mathrm{~kg}$ using Tanita weighing scales (TBF-410) and height was measured to the nearest $0.1 \mathrm{~cm}$ using a Leicester stadiometer. For both studies, BMI was calculated according to Quetelet's formula $\left(\mathrm{kg} / \mathrm{m}^{2}\right)$ and weight status (defined as two categories: normal weight; overweight/obese) was defined using the International Obesity Task Force BMI cut-offs ${ }^{(14)}$.

\section{Misreporting}

Among the NI adolescents, dietary intake by $7 \mathrm{~d}$ weighed dietary record was assessed concurrently with DLW measurements of TEE, as previously described ${ }^{(11)}$. In the main NDNS it was not feasible to undertake measures of TEE by DLW; therefore, the validity of the $7 \mathrm{~d}$ weighed dietary record was assessed against estimated energy requirements (EER) calculated from published sex and age-specific equations ${ }^{(15)}$. As previously described ${ }^{(16)}$, these equations are derived from DLW energy expenditure data and allow for four physical activity levels (sedentary, low activity, active and very active) with a corresponding activity coefficient in the EER equations. This has been demonstrated to be a valid measure of estimated energy expenditure in the NDNS of Young People ${ }^{(17)}$. In order to compare levels of misreporting of EI between the two studies, the EER method was also applied to the NI cohort.
Since TEE was measured by DLW in the NI cohort, the validity of EI data using the EER method was compared with the measured DLW TEE method. The EI:EER ratio measured by DLW and that estimated using the EER method were not significantly different (median 76.3 (interquartile range (IQR) 62.9, 89.4) and 77.1 (IQR 68.3, 93.6), respectively $P=0 \cdot 346$ ). The mean level of agreement between the two methods was 327.97 (95\% CI 4418.4, 3762.4), suggesting good agreement. Sensitivity of TEE estimated by the EER method compared with the gold standard DLW was high $(90 \%)$ and specificity also high $(85.7 \%)$. We therefore concluded that the EER method was a suitable approach for assessing misreporting of EI between adolescents in 1997 and those in 2005.

Given the daily variability in EI and energy expenditure at the individual level, confidence limits of agreement between reported EI and EER were calculated based on the number of days of dietary recording ${ }^{(18)}$.

$$
\mathrm{CV}_{\mathrm{t}}=\sqrt{\left(\mathrm{CV}_{\mathrm{EE}}^{2}+\mathrm{CV}_{\mathrm{EI}}^{2}\right) / \mathrm{d}}
$$

where $\mathrm{CV}_{\mathrm{t}}$ is the $\mathrm{CV}$ for both energy expenditure and $\mathrm{EI}, \mathrm{d}$ is the number of days of dietary recording and $\mathrm{CV}_{\mathrm{EE}}$ and $\mathrm{CV}_{\mathrm{EI}}$ are the $\mathrm{CV}$ for energy expenditure and EI respectively. The $\mathrm{CV}_{\mathrm{EI}}$ calculated from the NDNS and NI adolescents was 21.23 and 28.29 respectively. For both studies, the $\mathrm{CV}_{\mathrm{EE}}$ used was $8.2 \%$ based on DLW studies of $7 \mathrm{~d}$ duration ${ }^{(18)}$ since the EER equations were developed from DLW studies. This gave a total value $\left(\mathrm{CV}_{\mathrm{t}}\right)$ of $13.16 \%$ and $11.13 \%$ for the NDNS and NI adolescents respectively.

\section{Statistical analysis}

Data were assessed for normality using the KolmogorovSmirnov test for the NDNS study and the Shapiro-Wilk test for the NI study. Normally distributed data are shown as mean and standard deviations. Skewed data are shown as median and IQR. General characteristics were compared by MannWhitney U tests. Frequency of consumption of snack foods was compared by student's independent $t$ test. Portion sizes were compared by the Mann-Whitney $U$ test. Weight status was compared by $\chi^{2}$ tests. EI:EER was compared across portion sizes of selected food groups by one-way ANOVA. All statistical analyses were performed using SPSS (Statistical package for the social sciences, version 11.0.1; SPSS UK Ltd, Chertsey, UK) with a $P$ value of 0.05 used for the significance level.

\section{Results}

\section{General characteristics}

Anthropometry. The 2005 adolescents were significantly heavier and taller than their 1997 counterparts $(P=0.004$, $P<0.001$, respectively); however, neither BMI nor waist circumference was significantly different between the two groups (Table 1). Overall, a significantly lower proportion of the 2005 group was considered to be normal weight compared with the 1997 group $(P<0 \cdot 001)$. Correspondingly, a significantly higher proportion of the 2005 group compared with the 1997 group was considered to be overweight or obese $(P<0 \cdot 001)$. The 2005 boys were significantly taller and 
Table 1. General and dietary characteristics of National Diet and Nutrition Survey (NDNS) group (1997) compared with Northern Ireland (NI) group (2005)§ (Medians and interquartile ranges (IQR))

\begin{tabular}{|c|c|c|c|c|c|c|c|c|c|c|c|c|}
\hline & \multicolumn{4}{|c|}{ Total sample } & \multicolumn{8}{|c|}{ Sex differences } \\
\hline & \multicolumn{2}{|c|}{ NDNS 1997} & \multicolumn{2}{|c|}{$\mathrm{Nl}$ cohort 2005} & \multicolumn{4}{|c|}{ Boys } & \multicolumn{4}{|c|}{ Girls } \\
\hline & \multicolumn{2}{|c|}{434} & \multicolumn{2}{|r|}{47} & \multicolumn{2}{|c|}{$\begin{array}{c}\text { NDNS } \\
209\end{array}$} & \multicolumn{2}{|r|}{$\begin{array}{l}\mathrm{NI} \\
29\end{array}$} & \multicolumn{2}{|c|}{$\begin{array}{c}\text { NDNS } \\
225\end{array}$} & \multicolumn{2}{|c|}{$\begin{array}{l}\mathrm{NI} \\
18\end{array}$} \\
\hline & Median & IQR & Median & IQR & Median & IQR & Median & IQR & Median & IQR & Median & IQR \\
\hline \multicolumn{13}{|l|}{ General characteristics } \\
\hline Age (years) & 14.9 & $14,15 \cdot 9$ & $15 \cdot 0$ & 14,16 & 14.9 & $14,15 \cdot 9$ & $15 \cdot 0$ & $14,15 \cdot 5$ & 14.9 & 14,16 & $15 \cdot 0$ & 14,16 \\
\hline Weight $(\mathrm{kg})^{\star}, \dagger$ & 56.0 & $49 \cdot 3,64 \cdot 2$ & $62 \cdot 1$ & $52 \cdot 5,73 \cdot 7$ & 56.5 & $49 \cdot 4,65.9$ & $63 \cdot 1$ & $54 \cdot 5,74 \cdot 2$ & 54.7 & $49 \cdot 1,61 \cdot 1$ & 57.4 & $52 \cdot 4,66 \cdot 3$ \\
\hline Height $(m)^{\star}, \dagger$ & 1.63 & $1.57,1.69$ & 1.68 & $1.62,1.78$ & 1.67 & $1 \cdot 6,1 \cdot 7$ & 1.76 & $1.65,1.80$ & 1.61 & $1 \cdot 6,1 \cdot 66$ & 1.63 & $1.60,1 \cdot 70$ \\
\hline $\mathrm{BMI}\left(\mathrm{kg} / \mathrm{m}^{2}\right)$ & 20.6 & $18 \cdot 9,23 \cdot 1$ & 20.9 & $19 \cdot 3,23 \cdot 6$ & 20.4 & $18 \cdot 6,22 \cdot 7$ & 20.8 & $18.9,23.5$ & 21.0 & $19 \cdot 2,23 \cdot 3$ & 21.6 & $19 \cdot 6,24.5$ \\
\hline Normal weight \|| (\%)*,†,‡ & \multirow{2}{*}{\multicolumn{2}{|c|}{$77 \cdot 2$}} & \multirow{2}{*}{\multicolumn{2}{|c|}{74.5}} & \multirow{2}{*}{\multicolumn{2}{|c|}{77.5}} & \multirow{2}{*}{\multicolumn{2}{|c|}{$75 \cdot 9$}} & \multirow{2}{*}{\multicolumn{2}{|c|}{$76 \cdot 9$}} & \multirow{2}{*}{\multicolumn{2}{|c|}{72.2}} \\
\hline Overweight/obese \| $(\%)^{*}, \dagger, \ddagger$ & & & & & & & $24 \cdot 1$ & & & $18 \cdot 6$ & & $27 \cdot 8$ \\
\hline Waist $(\mathrm{cm})$ & 71.5 & $67 \cdot 3,77 \cdot 7$ & 69.0 & $69.0,77.4$ & 74.4 & $69 \cdot 6,82 \cdot 3$ & 74.0 & $69.0,80.5$ & $70 \cdot 0$ & $64 \cdot 9,740$ & 73.0 & $68 \cdot 7,77.0$ \\
\hline \multicolumn{13}{|l|}{ Dietary characteristics } \\
\hline El $(k J / d)^{\star}, t, \ddagger$ & 7954 & 6399,9253 & 9475 & 7677,12003 & 8933 & 7608,10387 & 11197 & 9305,12367 & 6904 & 5839,8261 & 7693 & 6513,9235 \\
\hline Eating occasions $/ d q^{\star}, \dagger, \ddagger$ & 8.6 & $7 \cdot 1,10 \cdot 4$ & 5.3 & $4 \cdot 4,6 \cdot 0$ & 8.7 & $7 \cdot 2,11 \cdot 1$ & 5.6 & $4 \cdot 6,7 \cdot 0$ & 8.4 & $6 \cdot 9,10 \cdot 1$ & 4.7 & $4 \cdot 2,5 \cdot 7$ \\
\hline$\%$ protein $/ \mathrm{d}^{\star \star}$ & 13.3 & $11 \cdot 9,14 \cdot 7$ & 13.9 & $12 \cdot 4,15 \cdot 8$ & 13.5 & $12 \cdot 2,14 \cdot 7$ & 13.9 & $12 \cdot 3,15 \cdot 7$ & $13 \cdot 2$ & $11 \cdot 8,14.7$ & 13.9 & $12 \cdot 5,16 \cdot 2$ \\
\hline$\%$ fat $/ d^{\star \star}$ & 34.8 & $31 \cdot 9,37 \cdot 8$ & 34.7 & $31 \cdot 4,37.9$ & 34.4 & $32 \cdot 3,37 \cdot 9$ & 33.8 & $31 \cdot 3,39 \cdot 4$ & 35.1 & $31 \cdot 7,37 \cdot 8$ & 35.5 & $31 \cdot 0,37 \cdot 7$ \\
\hline$\% \mathrm{CHO} / \mathrm{d}^{\star \star}$ & 54.5 & $51 \cdot 1,57 \cdot 9$ & 54.8 & $49 \cdot 5,57 \cdot 7$ & 55.0 & $51 \cdot 2,57 \cdot 8$ & 54.8 & $50 \cdot 3,57 \cdot 9$ & 54.1 & $50 \cdot 8,58 \cdot 1$ & 54.7 & $48 \cdot 2,57 \cdot 8$ \\
\hline$\%$ total sugars $/ \mathrm{d}^{\star \star}$ & $23 \cdot 1$ & $19 \cdot 3,27 \cdot 3$ & $23 \cdot 0$ & $18 \cdot 3,29 \cdot 6$ & 22.9 & $19 \cdot 3,27 \cdot 5$ & $23 \cdot 2$ & $17 \cdot 9,31 \cdot 7$ & $23 \cdot 1$ & $19 \cdot 2,27 \cdot 1$ & 22.9 & $20 \cdot 1,28 \cdot 4$ \\
\hline $\operatorname{EER}(\mathrm{kJ} / \mathrm{d})^{*}, \dagger$ & 10804 & 9896,12540 & 11868 & 10503,15006 & 12541 & 11307,14117 & 14063 & 12199,15577 & 10005 & 9535,10543 & 10371 & 9857,11029 \\
\hline El:EER ${ }^{\star}, \dagger$ & 0.69 & $0.58,0.84$ & 0.77 & $0.68,0.93$ & 0.71 & $0.58,0.85$ & 0.81 & $0.70,0.90$ & 0.69 & $0.60,0.80$ & 0.74 & $0.64,0.90$ \\
\hline
\end{tabular}

El, energy intake; $\mathrm{CHO}$ carbohydrate; $\mathrm{EER}$, estimated energy requirement.

Data were compared using the Mann-Whitney $U$ test $(P<0.05$ significant).

* Significant differences between total study groups $(P<0.01$, all cases $)$.

$\dagger$ significant differences observed between groups of boys $(P<0.01$, all cases $)$

f significant differences observed between groups for girls $(P<0.01$, all cases $)$.

For details of subjects and procedures, see Methods.

Derived from International Obesity Task Force cut-offs ${ }^{(14)}$ compared using $x^{2}$ tests.

1 Average number of eating occasions per $\mathrm{d}$ (total number of eating occasions/total number of recording days: $7 \mathrm{~d}$ for all NDNS participants and ranging from $5-7 \mathrm{~d}$ for NI participants)

${ }_{* \star}^{*}$ Expressed as percentage total energy intake. 
heavier than the 1997 boys; however, no differences in BMI or waist circumference were observed among the boys between groups. A significantly lower proportion of the 2005 boys were considered to be normal weight compared with the 1997 boys $(P<0 \cdot 001)$. Correspondingly, a significantly higher proportion of the 2005 boys compared with the 1997 boys were considered to be overweight or obese $(P<0.001)$. This was also the case for the 2005 girls compared with the 1997 girls; however, there were no differences in weight, height, BMI or waist circumference among the girls between groups.

\section{Dietary characteristics}

Absolute EI was significantly higher among the 2005 adolescents compared with the 1997 adolescents, overall, in the 2005 boys compared with the 1997 boys and the 2005 girls compared with the 1997 girls $(P<0.001$ in all cases) (Table 1). No significant differences in \% contribution of macronutrients to EI were observed between the two groups overall or among boys or girls between groups. The overall average number of eating occasions per d was significantly lower in the 2005 group compared with the 1997 group, and among the 2005 boys compared with the 1997 boys and the 2005 girls compared with the 1997 girls.

\section{Misreporting}

Compared with the NDNS adolescents, EER were significantly higher $(P=0.001)$ among the NI adolescents overall and among the NI boys compared with the NDNS boys due to their increased body size (Table 1). This, in part, explains the significantly higher EI observed among the NI adolescents compared with the NDNS adolescents. Overall, the ratio of EI:EER was significantly lower among the 1997 group compared with the 2005 group (median $69 \%$ and $77 \%$ respectively, $P=0.006$ ) and among the 1997 boys compared with the 2005 boys $(71 \%$ and $81 \%$ respectively, $P<0.05)$, suggesting greater misreporting of EI by the 1997 NDNS adolescents, driven by the boys. No differences in EI:EER between the 1997 girls and the 2005 girls were observed. Overall, EI:EER was significantly lower among the overweight/obese adolescents compared with the normal weight adolescents in both the 1997 group $(61.3 \%$ and $72.4 \%$, respectively; $P<0.001)$ and the 2005 group $(70.8 \%$ and $81.6 \%$ respectively; $P=0.018$ ), suggesting greater misreporting among the overweight/obese adolescents in both groups (data not shown). Based on confidence limits of agreement between reported EI and $\mathrm{EER}^{(18)}$, the overall percentage of adolescents identified as under reporters was 82.7 in 1997 and $70 \cdot 2$ in 2005 .

\section{Snack intake}

Median snack EI was significantly higher among the 2005 adolescents compared with the 1997 adolescents $(P<0 \cdot 001)$ (Table 2). Despite this, overall average number of snackeating occasions was significantly lower in the 2005 cohort compared with the 1997 group and among both the 2005 boys compared with the 1997 boys and the 2005 girls compared with the 1997 girls. No differences in \% contribution of snacks to EI, or in \% contribution of macronutrients from snacks to EI were observed between the two groups as a whole or when stratified by sex. No differences in snack EI, $\%$ energy from snacks, frequency of snack food consumption or \% contribution of macronutrients from snacks to EI were observed between normal weight and overweight/obese adolescents in either group (data not shown).

\section{Types of snack foods consumed (data not shown)}

Carbonated and soft drinks were the most commonly consumed snack food by the NDNS adolescents in 1997 (consumed by $85 \%$ of participants) and this was also the case among the NI adolescents in 2005 (consumed by $87 \%$ of participants). Low-energy versions of carbonated and soft drinks were consumed by $46 \%$ of the 1997 adolescents and by only $14 \%$ of the 2005 adolescents. While milks and beverages were the second and third most popular snack food choice among the NDNS adolescents in 1997 (consumed by $84 \%$ and $82 \%$ of participants, respectively), chocolate confectionery and bread were the second and third choice among the NI adolescents in 2005 (consumed by $79 \%$ and $77 \%$ of participants, respectively). Other foods that were more commonly consumed as snacks by the adolescents in 2005 than those in 1997 were biscuits, breakfast cereals, buns, cakes and pastries and fruit. Foods that were less commonly consumed as snacks by the adolescents in 2005 compared with those in 1997 were fat spreads and oils, potato and potato products, vegetable and vegetable dishes, sugars, syrups and preserves and soups, sauces and condiments.

\section{Comparison of frequency of consumption and portion size of top fifteen snacks}

Table 3 compares snack-eating occasions per $\mathrm{d}$ and portion sizes consumed of the top fifteen snack foods between the NDNS adolescents in 1997 and the NI adolescents in 2005. A trend for a higher number of snacking occasions of carbonated and soft drinks in the 2005 group than in the 1997 group was observed. When consumers of low-energy versions of carbonated and soft drinks were removed from the analysis (46\% in 1997 and $14 \%$ in 2005), this trend became significant, with $27 \%$ more snacking occasions of high-energy carbonated and soft drinks observed among the 2005 adolescents compared with the 1997 adolescents $(0.73$ times per $d$ compared with 0.53 times per d respectively; $P=0.014$ ). Furthermore, portion size of all carbonated and soft drinks was found to be significantly higher in the 2005 group compared with the 1997 group $(P<0.001)$ and this result remained significant when low-energy drink consumers were removed from the analysis $(P=0.02)$. In order to assess the extent to which water intake (as a snack) in the 2005 group (consumed by $23 \%$ of participants either as a drink or a dilutent for soft drinks) may have determined the increased portion size observed of carbonated and soft drinks in the 2005 group compared with the 1997 group, 'water' consumers were excluded from the 2005 group and the difference in portion size of 'carbonated and soft drinks' between the two groups was re-examined (data not shown). The significantly higher portion size of carbonated and soft drinks observed in the 2005 group compared with the 1997 group remained following this analysis (median portion size of $269 \mathrm{~g}$ in the 
2005 group (value before water exclusion $295 \mathrm{~g}$ ) compared with $238 \mathrm{~g}$ in the 1997 group; $P=0 \cdot 002$ ). In the case of 'beverages', for the 1997 group, water was included both as a dilutent for powdered drinks and essences and as a drink (i.e. tap water); whereas for the 2005 group, water was only included as a dilutent for powdered drinks and essences (referred to as 'powdered drinks and beverages made up with water'). Thus, we are satisfied that both the increases in portion size observed for "carbonated and soft drinks' and for 'beverages' over the 8-year period are irrespective of water intake in the 2005 cohort. Snacking occasions of beverages were $44.6 \%$ lower among the 2005 adolescents compared with the 1997 adolescents (from 0.74 times/d to 0.41 times/d respectively, $P<0.001$ ). Interestingly, however, the portion size of beverages was $20.4 \%$ higher among the 2005 adolescents compared with the 1997 adolescents $(P=0 \cdot 007)$. Only $29 \%$ ( $n 125)$ of the 1997 cohort reported consumption of tea/coffee as a snack compared with $64 \%$ ( $n$ 30) of the 2005 group. Therefore, the increased portion size of 'beverages' observed between the two groups could also potentially have been driven by the addition of milk in tea and coffee by the 2005 group. In order to address this possibility, tea and coffee were excluded from both groups (both with and without the addition of milk in the 2005 group) and the difference in portion size of 'beverages' was re-examined between the two groups. Portion size of 'beverages' was still found to be significantly higher in the 2005 group (median $216 \mathrm{~g}(n$ 17) $v .180 \mathrm{~g}$ (n 309), $P=0 \cdot 017$, data not shown) following this analysis. The portion sizes of milks, crisps and savoury snacks and breakfast cereals were also significantly higher among the adolescents in 2005 compared with those in $1997(P<0.001, P=0.015$, $P=0 \cdot 036$. respectively) with no change in number of snacking occasions observed.

While frequency of consumption of vegetables and vegetable dishes was significantly lower among the 2005 adolescents compared with the 1997 adolescents $(P=0.003)$, no difference in portion size between groups was observed. No significant differences in frequency of consumption of fruit or in portion size of fruit consumed were observed between the two groups.

\section{Differences in portion size of selected food groups according} to sex

Portion sizes consumed of carbonated and soft drinks (both with and without low-energy drink consumers), milks, beverages, crisps and savoury snacks and breakfast cereals were significantly higher among the 2005 adolescents compared with the 1997 adolescents overall and among the 2005 boys compared with the 1997 boys. Only the portion size of milks was found to be significantly higher in the girls in the 2005 NI cohort compared with the girls in the 1997 NDNS group (Table 4).

\section{Differences in portion size of selected food groups according to weight status}

Portion sizes consumed of carbonated and soft drinks, milks, beverages, crisps and savoury snacks and breakfast cereals were compared between normal weight and overweight/ obese adolescents in both groups (Table 5). No differences in the portion size of any of these food groups were observed 
Table 3. Changes in adolescent snacking habits between 1997 and $2005^{\star}, \dagger$

(Means and standard deviations or medians and interquartile ranges (IQR))

\begin{tabular}{|c|c|c|c|c|c|c|c|c|c|c|}
\hline & \multicolumn{5}{|c|}{ Snack-eating occasions/dł } & \multicolumn{5}{|c|}{ Portion size (g/eating occasion)§ } \\
\hline & \multicolumn{2}{|c|}{$\begin{array}{l}\text { NDNS } \\
(1997)\end{array}$} & \multicolumn{2}{|c|}{$\begin{array}{l}\text { NI cohort } \\
\text { (2005) }\end{array}$} & \multirow[b]{2}{*}{$P$-value } & \multicolumn{2}{|c|}{$\begin{array}{l}\text { NDNS } \\
(1997)\end{array}$} & \multicolumn{2}{|c|}{$\begin{array}{l}\text { NI cohort } \\
\text { (2005) }\end{array}$} & \multirow[b]{2}{*}{$P$-value } \\
\hline & Mean & SD & Mean & SD & & Median & IQR & Median & IQR & \\
\hline All carbonated and soft drinks\| & 0.68 & 0.52 & 0.76 & 0.49 & 0.366 & 226 & 164,293 & 284 & 230,430 & $<0.001$ \\
\hline Non-diet carbonated and soft drinks $\uparrow$ & 0.53 & 0.47 & 0.73 & 0.49 & 0.014 & 238 & 168,326 & 295 & 220,429 & 0.022 \\
\hline Milks ${ }^{\star \star}$ & 0.62 & 0.49 & 0.52 & 0.32 & 0.098 & 120 & 62,197 & 232 & 185,318 & $<0.001$ \\
\hline Beveragest† & 0.74 & 0.56 & 0.41 & 0.33 & $<0.001$ & 187 & 142,230 & 234 & 185,293 & 0.007 \\
\hline Breads & 0.40 & 0.28 & 0.43 & 0.30 & 0.663 & 67 & 53,86 & 66 & 53, 82 & 0.989 \\
\hline Meat and meat dishes & 0.37 & 0.24 & 0.39 & 0.25 & 0.590 & 100 & 62,45 & 117 & 67,219 & 0.118 \\
\hline Fat spreads and oils & 0.35 & 0.23 & 0.39 & 0.30 & 0.449 & 10 & 8,14 & 12 & 7,16 & 0.703 \\
\hline Chocolate confectionary & 0.35 & 0.27 & 0.40 & 0.31 & 0.353 & 43 & 30,54 & 37 & 28,52 & 0.342 \\
\hline Crisps and savoury snacks & 0.35 & 0.25 & 0.43 & 0.36 & 0.225 & 27 & 25,30 & 31 & 25,35 & 0.015 \\
\hline Potato and potato products & 0.26 & 0.17 & 0.23 & 0.11 & 0.289 & 153 & 101,200 & 165 & 120,180 & 0.821 \\
\hline Vegetables and vegetable dishes & 0.30 & 0.22 & 0.22 & 0.10 & 0.003 & 60 & 37,100 & 66 & 48,80 & 0.643 \\
\hline Sugars, syrups and preserves & 0.48 & 0.40 & 0.33 & 0.26 & 0.042 & 11 & 6,17 & 12 & 8,20 & 0.248 \\
\hline Biscuits & 0.33 & 0.24 & 0.33 & 0.25 & 0.909 & 29 & 22,39 & 31 & 20,43 & 0.989 \\
\hline Soups, sauces and condiments & 0.38 & 0.39 & 0.24 & 0.17 & 0.004 & 30 & 8,96 & 17 & 12,55 & 0.762 \\
\hline Breakfast cerealł‡ & 0.30 & 0.23 & 0.32 & 0.21 & 0.680 & 50 & 37,38 & 60 & 41,105 & 0.036 \\
\hline Fruit & 0.32 & 0.23 & 0.37 & 0.21 & 0.341 & 112 & 89,136 & 110 & 100,138 & 0.650 \\
\hline Buns, cakes and pastries & 0.28 & 0.24 & 0.32 & 0.22 & 0.497 & 62 & 45,83 & 75 & 43,126 & 0.240 \\
\hline
\end{tabular}

*Food groups shown were ranked in descending order of percentage consumers among National Diet and Nutrition Survey (NDNS) adolescents aged 13-16 years.

†For details of subjects and procedures, see Methods.

† Differences were assessed by independent $t$ test $(P<0.05$, significant)

$\S$ Differences were assessed by the Mann-Whitney $U$ test $(P<0.05$, significant)

II NDNS group: all concentrated, carbonated and ready to drink soft drinks (both diet and non-diet) and water as a dilutent only; Northern Ireland (NI) cohort: concentrated and ready to drink soft drinks (both diet and non-diet), water as a dilutent and/or water as a drink.

I NDNS group: non-diet concentrated, carbonated and ready to drink soft drinks consumers only (46\%); NI group: non-diet concentrated, carbonated and ready to drink soft drinks consumers only (14\%).

** Whole milk, semi-skimmed milk, skimmed milk, UHT milk in both datasets.

††NDNS group: tea (infusion), coffee (infusion), fruit juice, powdered drinks and essences, tap water (as a drink) and water as a dilutent for powdered drinks; NI cohort: tea (infusion, with milk), coffee (infusion, with milk), fruit juice and powdered drinks and essences (made up with water).

㧊 Not inclusive of milk in either dataset.

according to weight status in the NDNS group; however, the portion size of beverages was found to be significantly higher among the overweight/obese adolescents compared with the normal weight adolescents in the NI cohort (median (g/d) 289 (IQR 249, 356) v. 193 (IQR 170, 267); $P=0 \cdot 018)$. Despite this finding, beverages were consumed significantly more frequently by the normal weight adolescents than the overweight/obese adolescents in the NI cohort (data not shown: 0.47 times per d compared with 0.25 times per d; $P=0.04)$. No differences in frequency of these food groups were observed according to weight status in the NDNS group.

\section{Misreporting}

Given the extent of under-reporting in both cohorts, EI from snacks was examined in both the NDNS adolescents and the NI adolescents both above and below the median of EI:EER; no differences were observed in either group (data not shown). In addition, the ratio of EI:EER was compared across quartiles of portion size of carbonated and soft drinks, beverages, milks, crisps and savoury snacks and breakfast cereals (data not shown). In the 1997 NDNS group, as portion size of all food groups increased, no significant differences in EI:EER were observed. In the 2005 NI cohort, however, EI:EER was significantly lower in the second highest portion size quartile of carbonated and soft drinks compared with the highest quartile. No further differences in EI:EER according to quartiles of portion size of the remaining food groups were observed in the NI group.

\section{Discussion}

The aim of the current analysis was to compare snack food patterns (in terms of types of foods consumed, portion size, frequency and percentage consumers) among UK adolescents studied in 1997 to those among adolescents studied 8 years later in 2005. Overall, the most important changes in snack food patterns over the 8-year time period were in relation to EI and portion size. Specifically, total EI was significantly higher among the 2005 adolescents compared with the 1997 adolescents, due in part to the increased energy requirements among the 2005 adolescents as a result of being taller and heavier than their 1997 counterparts. Consequently, EI from snacks was significantly higher in the 2005 cohort and, although not significant, a trend for a higher percentage EI from snacks in the 2005 cohort compared with the 1997 group was observed. Carbonated and soft drinks remained the most popular choice of snack over this 8-year period; however, the portion size consumed and frequency of consumption were significantly higher among the adolescents in 2005 compared with those in 1997. In addition, despite a lower frequency of consumption of both milks and beverages, the portion size of both was significantly higher among the adolescents in 2005 compared with those in 1997. 
Table 4. Differences in portion size* of selected food groups according to sex $\uparrow$

(Medians and interquartile ranges (IQR))

\begin{tabular}{|c|c|c|c|c|c|c|c|c|c|c|}
\hline & \multicolumn{4}{|c|}{ Boys } & \multirow[b]{3}{*}{$P$-value } & \multicolumn{4}{|c|}{ Girls } & \multirow[b]{3}{*}{$P$-value } \\
\hline & \multicolumn{2}{|c|}{$\begin{array}{c}\text { NDNS } 1997 \\
209\end{array}$} & \multicolumn{2}{|c|}{$\begin{array}{c}\mathrm{NI} 2005 \\
29\end{array}$} & & \multicolumn{2}{|c|}{$\begin{array}{c}\text { NDNS } 1997 \\
225\end{array}$} & \multicolumn{2}{|c|}{$\begin{array}{c}\text { NI } 2005 \\
18\end{array}$} & \\
\hline & Median & IQR & Median & IQR & & Median & IQR & Median & IQR & \\
\hline All carbonated and soft drinks $\ddagger$ & 239 & 184,298 & 344 & 260,448 & 0.012 & 205 & 157,283 & 247 & 214,363 & 0.059 \\
\hline Non-diet carbonated and soft drinks§ & 258 & 200,300 & 344 & 268,448 & 0.001 & 210 & 143,309 & 243 & 196,363 & 0.093 \\
\hline Milks\| & 148 & 78,224 & 273 & 194,359 & $<0.001$ & 99 & 53,176 & 217 & 100,244 & 0.005 \\
\hline Beverages $\rrbracket$ & 196 & 144,238 & 256 & 190,321 & 0.011 & 179 & 137,225 & 193 & 170,274 & 0.271 \\
\hline Crisps and savoury snacks & 27 & 25,30 & 31 & 26,38 & 0.016 & 28 & 25,30 & 31 & 22,35 & 0.351 \\
\hline Breakfast cereals ${ }^{\star \star}$ & 56 & 39,72 & 69 & 47,115 & 0.023 & 47 & 35,58 & 37 & 26,56 & 0.403 \\
\hline
\end{tabular}

Differences were assessed by the Mann-Whitney $U$ test $(P<0.05$, significant).

${ }^{*}$ Refers to $g$ per eating occasion.

†For details of subjects and procedures, see Methods.

$¥$ National Diet and Nutritional Survey (NDNS) group: all concentrated, carbonated and ready to drink soft drinks (both diet and non-diet) and water as a dilutent only; Northern Ireland (NI) cohort: concentrated and ready to drink soft drinks (both diet and non-diet), water as a dilutent and/or water as a drink.

$\S$ NDNS group: non-diet concentrated, carbonated and ready to drink soft drinks consumers only (46\%); NI group: non-diet concentrated, carbonated and ready to drink soft drinks consumers only $(14 \%)$.

\| Whole milk, semi-skimmed milk, skimmed milk, UHT milk in both datasets.

I NDNS group: tea (infusion), coffee (infusion), fruit juice, powdered drinks and essences, tap water (as a drink) and water as a dilutent for powdered drinks. NI cohort: tea (infusion, with milk), coffee (infusion, with milk), fruit juice and powdered drinks and essences (made up with water).

${ }^{* *}$ Not inclusive of milk in either dataset.

Interestingly, $46 \%$ of the 1997 group reported consumption of low-energy versions of carbonated and soft drinks compared with only $14 \%$ of the 2005 group. In addition to the increased portion size observed, frequency of consumption of high-energy carbonated and soft drinks was significantly higher among the 2005 adolescents compared with the 1997 adolescents. The higher consumption of sugarsweetened beverages has been postulated to play an important role in excessive weight gain among children and adolescents $^{(19,20)}$. The exact mechanism is not well understood, but is thought to be attributed to factors including increased $\mathrm{EI}^{(21)}$ and blunted satiety in response to liquid energy, thus facilitating excess consumption ${ }^{(22)}$. In support of this,
EI, body weight and fat mass have been reported to have increased in overweight adults following a 10-week intervention with sucrose-sweetened beverages and foods. Of note, $70 \%$ of the sucrose came from beverages ${ }^{(23)}$. A recent study, however, found no evidence that overweight children who participated in the NDNS (1997) derived a greater proportion of their energy from sugar-sweetened soft drinks compared with lean children ${ }^{(24)}$. In the current analysis, while overweight/obese adolescents in 2005 consumed a greater portion size of beverages (inclusive of tea, coffee and fruit juice) compared with their normal weight counterparts, no differences in portion size of high-energy carbonated and soft drinks were observed between normal weight and

Table 5. Portion size* of selected food groups according to weight status (Medians and interquartile ranges (IQR))

\begin{tabular}{|c|c|c|c|c|c|c|c|c|c|c|}
\hline \multirow[b]{3}{*}{ Weight status $\ddagger$} & \multicolumn{4}{|c|}{ NDNS group 1997} & \multirow[b]{3}{*}{$P$-value } & \multicolumn{4}{|c|}{ NI cohort 2005} & \multirow[b]{3}{*}{$P$-value } \\
\hline & \multicolumn{2}{|c|}{$\begin{array}{l}\text { Normal weight } \\
\qquad(n 335)\end{array}$} & \multicolumn{2}{|c|}{$\begin{array}{l}\text { Overweight/obese } \\
\qquad(n 88)\end{array}$} & & \multicolumn{2}{|c|}{$\begin{array}{l}\text { Normal weight } \\
\qquad(n 35)\end{array}$} & \multicolumn{2}{|c|}{$\begin{array}{l}\text { Overweight/obese } \\
\qquad(n 12)\end{array}$} & \\
\hline & Median & IQR & Median & IQR & & Median & IQR & Median & IQR & \\
\hline All carbonated and soft drinks§ & 219 & 162,289 & 242 & 184,313 & 0.178 & 314 & 233,440 & 272 & 219,401 & 0.495 \\
\hline Non diet carbonated \& soft drinks\| & 231 & 164,323 & 261 & 194,335 & 0.098 & 326 & 240,440 & 268 & 200,401 & 0.301 \\
\hline Milks ${ }^{\star *}$ & 121 & 64,194 & 121 & 57,226 & 0.813 & 227 & 183,305 & 249 & 170,401 & 0.594 \\
\hline Crisps and savoury snacks & 28 & 25,30 & 27 & 25,30 & 0.853 & 31 & 27,36 & 28 & 22,35 & 0.503 \\
\hline Breakfast cereals $†$ & 50 & 37,67 & 53 & 38,79 & 0.316 & 60 & 41,100 & 60 & 30,114 & 0.646 \\
\hline
\end{tabular}

Data were compared using the Mann-Whitney $U$ test $(P<0.05$ significant).

${ }^{*}$ Refers to g per eating occasion.

$\dagger$ For details of subjects and procedures, see Methods.

$\ddagger$ Derived from International Obesity Task Force cut-offs ${ }^{(14)}$

§National Diet and Nutrition Survey (NDNS) group: all concentrated, carbonated and ready to drink soft drinks (both diet and non-diet) and water as a dilutent only;

Northern Ireland (NI) cohort: concentrated and ready to drink soft drinks (both diet and non-diet), water as a dilutent and/or water as a drink.

|| NDNS group: non-diet concentrated, carbonated and ready to drink soft drinks consumers only ( $46 \%)$; NI group: non-diet concentrated, carbonated and ready to drink soft

drinks consumers only (14\%).

I Whole milk, semi-skimmed milk, skimmed milk, UHT milk in both datasets.

${ }^{\star}$ NDNS group: tea (infusion), coffee (infusion), fruit juice, powdered drinks and essences, tap water (as a drink) and water as a dilutent for powdered drinks; NI cohort: tea (infusion, with milk), coffee (infusion, with milk), fruit juice and powdered drinks and essences (made up with water).

†† Not inclusive of milk in either dataset. 
overweight/obese adolescents in either group. Even so, the current analysis shows that regardless of weight status, adolescents are an important target group for policy interventions aimed at reducing consumption of high-energy drinks.

Snack food intake, including EI and frequency of snack food consumption did not differ between normal weight adolescents and overweight/obese adolescents in either the 1997 group or the 2005 cohort in the current study. However, as significantly greater misreporting among the overweight/obese adolescents compared with their normal weight counterparts was observed in both groups, snack food intake among the overweight/obese adolescents may have been underestimated in both groups. Indeed, there is no doubt that extent of under-reporting is a major confounder in any studies involving the analysis of food intake ${ }^{(25)}$. Substantial under-reporting in the total NDNS group of young people aged 4-18 years has previously been reported ${ }^{(17)}$. The current study observed greater misreporting in the NDNS adolescents in 1997 compared with the NI adolescents in 2005. The extent of underreporting did not differ by sex in either group; however, we observed greater misreporting among the boys in the 1997 group compared with those in the 2005 cohort.

Given the extent of under-reporting in the 1997 NDNS, EI from snacks in the current study may have been underestimated more in the 1997 group than in the 2005 cohort. If the frequency of snack consumption was misreported more in 1997 than in 2005, this would mask a greater difference in the reduction of snacking frequency between the groups. However, if portion size was misreported more in 1997 than in 2005, the increase in portion size that we observed for specific snack foods may have been more apparent than real. We observed that as portion size of milks, beverages, crisps and savoury snacks and breakfast cereals increased within both groups in the current study, no differences in mean EI:EER in either group were observed. This suggests that the increased portion size observed of these specific food groups among the 2005 group was not driven by the level of under-reporting in the 1997 group. In the case of carbonated and soft drinks, no differences in EI:EER were observed as portion size increased in the NDNS group. However, in the 2005 adolescents, EI:EER was significantly lower in the second-highest quartile of portion size of carbonated and soft drinks, thus suggesting that the portion size of carbonated and soft drinks was misreported more among the 2005 group. If this was the case, then the increased portion size of carbonated and soft drinks observed over time was not overestimated, but in fact may have been underestimated, thus attenuating the result. These findings also suggest, however, that if consumption of certain food groups, in this case carbonated and soft drinks, is more commonly under-reported than others, assessment of specific food group consumption may be particularly problematic. Furthermore, since it is only possible to estimate the overall misreporting of EI, it is not clear whether misreporting affects the reporting of frequency of consumption and portion size equally or disproportionately.

Given that the adolescents in the current study originated from two different geographical regions (Britain and NI), it is possible that the findings were more attributable to geographical location differences than to a change over time. It was beyond the scope of the current research to fully address this issue, thereby limiting the interpretation of the findings.
Methodological differences, specifically at the level of food intake data coding and input, may also have played an important role in determining the current findings. For example, the way in which water was coded within the food group 'carbonated and soft drinks' was different between the two groups (in the 1997 group, water was included as a dilutent only; whereas for the 2005 group, 'water as a dilutent' or 'water as a drink' were indistinguishable). On exclusion of water consumers from the 2005 group, however, the portion size of carbonated and soft drinks was still significantly higher in the 2005 group. In the case of 'beverages', tea and coffee consumed were inclusive of milk in the 2005 group, but exclusive of milk in the 1997 group, which may have influenced the increased portion size of 'beverages' observed in the 2005 group. On excluding tea and coffee consumers from both groups, however, the portion size of beverages was still significantly higher in the 2005 group. Nevertheless, as both this analysis and that previously relating to water required exclusion of a substantial proportion of the 2005 group $(65 \%$ and $25 \%$ respectively), it is difficult to draw meaningful conclusions from either result. Given that milk added to tea and coffee was not included in the food group 'milks' for the 2005 group, we are satisfied that the increased portion size of 'milks' observed in the 2005 group is reflective of an increase in milk as a drink by itself or that added to breakfast cereal (given the increased portion size of breakfast cereal in the 2005 cohort, it may be the latter).

Additional limitations of the current analyses include the small sample size of the NI cohort and the arbitrary approach to defining a snack. Indeed, the lack of consensus about the way in which a snack is defined is currently a limitation in many studies, as different research outcomes could be invoked depending on the method used. The current study, like others ${ }^{(8,9)}$, used time of day to distinguish meals from snacks, in that food consumption, which took place at a recognised meal time within the structure of the day, was defined as a meal, while all other food consumption was classified as a snack. Uniquely though, the specific time frames used in the current study were chosen on the basis that they were broadly comparable to those generated from the original dietary data of the NI adolescents, in which eating occasions were self-defined; arguably, the most valid approach for accurately defining an eating occasion.

In conclusion, this is the first study to have provided information on the types of foods that are typically consumed as snacks by UK adolescents. The differences observed between the NDNS group in 1997 and the NI cohort in 2005 may be attributable to a change over time and/or geographical differences. In any case, from a public health perspective, the current findings provide scope for policy interventions to place particular emphasis on reducing typical portion sizes consumed of popular snack choices among UK adolescents.

\section{Acknowledgements}

The NI cohort study was funded by the Food Standards Agency, UK. There are no conflicts of interest. We thank the NI subjects for their willingness to participate in the study. K. L. R. is currently employed by Unilever, but at the time of the NI study was employed by the University of Ulster. 


\section{References}

1. McPherson K, Marsh T \& Brown M (2007) Modelling future trends in obesity and the impact on health. Foresight tackling obesities: Future choices. http://www.foreslight.gov.uk

2. Jebb SA, Rennie KL \& Cole TJ (2004) Prevalence of overweight and obesity among young people in Great Britain. Public Health Nutr 7, 461-465.

3. Zizza C, Siega-Riz AM \& Popkin BM (2001) Significant increase in young adults' snacking between 1977-1978 and 1994-1996 represents a cause for concern! Prev Med 32, 303-310.

4. de Graaf C (2006) Effects of snacks on energy intake: an evolutionary perspective. Appetite 47, 18-23.

5. Jahns L, Siega-Riz AM \& Popkin BM (2001) The increasing prevalence of snacking among US children from 1977 to 1996. J Pediatr 138, 493-498.

6. Sebastian R, Cleveland L, Goldmad J \& Moshfegh A (2007) Snacking behaviour of children and teenagers in the United States. FASEB 20, A189-A190.

7. Nielsen SJ \& Popkin BM (2003) Patterns and trends in food portion sizes, 1977-1998. JAMA 289, 450-453.

8. Ovaskainen ML, Reinivuo H, Tapanainen H, Hannila ML, Korhonen T \& Pakkala H (2006) Snacks as an element of energy intake and food consumption. Eur J Clin Nutr 60, 494-501.

9. Summerbell CD, Moody RC, Shanks J, Stock MJ \& Geissler C (1995) Sources of energy from meals versus snacks in 220 people in four age groups. Eur J Clin Nutr 49, 33-41.

10. Gregory J \& Lowe S (2000) National Diet and Nutrition Survey: Young People Aged 4 to 18 Years, Report of the Diet and Nutrition Survey, vol. 1. London: Stationery Office.

11. McGloin AF, Livingstone MB, Greene LC, et al. (2002) Energy and fat intake in obese and lean children at varying risk of obesity. Int J Obes Relat Metab Disord 26, 200-207.

12. Bates CJ, Prentice AM, Jackson LV, Smithers G, Wenlock R \& Farron M (2002), Appendix C. Feasibility Study Report. The National Diet and Nutrition Survey: Young People Aged 4-18 Years, Volume 1: Report of the Diet and Nutrition Survey. London: Social Survey Division of the Office of National Statistics.

13. Food Standards Agency (2002) McCance and Widdowson's The Composition of Foods, Sixth summary edition. Cambridge: Royal Society of Chemistry.
14. Cole TJ, Bellizzi MC, Flegal KM \& Dietz WH (2000) Establishing a standard definition for child overweight and obesity worldwide: international survey. Br Med J 320, 1240-1243.

15. Institute of Medicine of the National Academies (2002) Dietary Reference Intakes for Energy, Carbohydrate, Fiber, Fat, Fatty Acids, Cholesterol, Protein, and Amino Acids. Washington, DC: National Academies Press.

16. Rennie KL, Coward A \& Jebb SA (2007) Estimating underreporting of energy intake in dietary surveys using an individualised method. Br J Nutr 97, 1169-1176.

17. Rennie KL, Jebb SA, Wright A \& Coward WA (2005) Underreporting of energy intake in the National Diet and Nutrition Survey of young people. Br J Nutr 93, 241-247.

18. Black AE \& Cole TJ (2000) Within- and between-subject variation in energy expenditure measured by the doubly-labelled water technique: implications for validating reported dietary energy intake. Eur J Clin Nutr 54, 386-394.

19. Nielsen SJ, Siega-Riz AM \& Popkin BM (2002) Trends in food locations and sources among adolescents and young adults. Prev Med 35, 107-113.

20. Berkey CS, Rockett HR, Field AE, Gillman MW \& Colditz GA (2004) Sugar-added beverages and adolescent weight change. Obes Res 12, 778-788.

21. Malik VS, Schulze MB \& Hu FB (2006) Intake of sugar-sweetened beverages and weight gain: a systematic review. Am J Clin Nutr 84, 274-288.

22. DiMeglio DP \& Mattes RD (2000) Liquid versus solid carbohydrate: effects on food intake and body weight. Int $J$ Obes Relat Metab Disord 24, 794-800.

23. Raben A, Vasilaras TH, Moller AC \& Astrup A (2002) Sucrose compared with artificial sweeteners: different effects on ad libitum food intake and body weight after $10 \mathrm{wk}$ of supplementation in overweight subjects. Am J Clin Nutr 76, $721-729$.

24. Gibson S \& Neate D (2007) Sugar intake, soft drink consumption and body weight among British children: further analysis of National Diet and Nutrition Survey data with adjustment for under-reporting and physical activity. Int J Food Sci Nutr 58, $445-460$.

25. Livingstone MB \& Black AE (2003) Markers of the validity of reported energy intake. J Nutr 133, Suppl. 3, 895S-920S. 